\title{
Incorporating new parameters into pathological staging of RCC
}

The pathological features of renal cell carcinoma (RCC) are used to stage the tumor, and as prognostic indicators. Tumor invasion into the surrounding fat tissue and extension into the venous system has been associated with poor prognosis in patients with RCC. Findings from two new studies performed by a group based in Italy indicate that predictions of survival in patients with RCC and vascular invasion would be improved by assessing the extent of tumor fat invasion and the consistency of the venous tumor thrombus in histopathological investigations.

For their first report, Roberto Bertini and colleagues reviewed the pathological features of renal tumors in 174 patients with RCC and vascular invasion who had undergone radical nephrectomy and thrombectomy. The consistency of the tumor thrombus was graded as either solid ( $n=107$; a compact and cohesive growth, sometimes with a pseudocapsulelike endothelial lining) or friable ( $n=67$; a noncohesive growth lacking a pseudocapsule with tumor cells mixed with necrosis and fibrin). Thrombus consistency was found to be an important indicator of survival in patients with RCC. Cancer-specific survival during follow-up was only 8 months among patients with a friable thrombus compared with
55 months in those with a solid thrombus. Moreover, patients with a friable thrombus were at an increased risk of metastases, high tumor grade, high pathological stage and perinephric fat invasion $(P<0.05)$. Exactly why the presence of a friable thrombus contributes to disease progression is unknown and warrants further study. Bertini and co-workers postulate that a friable thrombus could be associated with a high risk of hematogenous spread of the tumor and an increased risk of systemic progression.

The effects of tumor fat invasion on patient survival were investigated in the team's second retrospective analysis, this time of 184 patients with RCC and venous tumor thrombosis. The researchers found that patients who had tumor invasion into the renal fat compartments were at an increased risk of cancer-specific mortality compared with those without tumor fat invasion. Presence of either perinephric fat invasion or renal sinus fat invasion (or both) was linked to tumor metastases, high tumor grade and increased tumor dimensions. Risk of death from cancer was two times higher in individuals with tumor fat invasion (either extension into the perinephric or renal sinus fat) than in those without. Moreover, those with both perinephric and renal sinus fat invasion were at the greatest risk of death

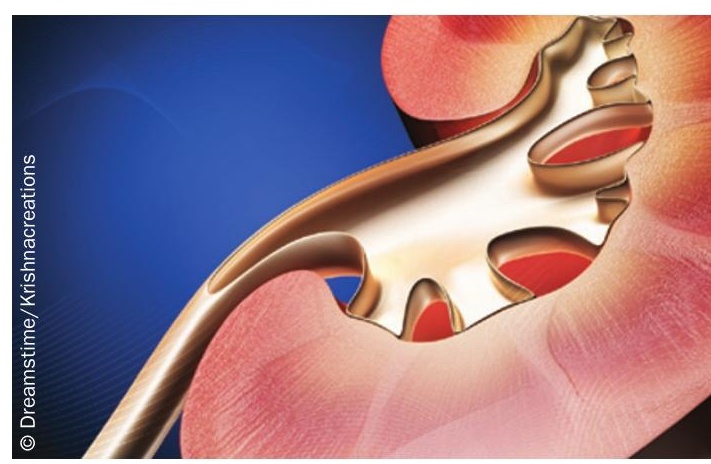

from cancer (threefold higher than those without tumor fat invasion) with only $20 \%$ of these patients surviving at 5 years (compared with $75 \%$ of those without tumor fat invasion).

Despite the retrospective nature of both studies, the authors conclude that both thrombus consistency and the extent of tumor fat invasion should be included in routine pathological investigations for RCC, as this could help to identify high-risk patients.

Katrina Ray

Original articles Bertini, R. et al. Impact of venous tumour thrombus consistency (solid vs friable) on cancer-specific survival in patients with renal cell carcinoma. Eur. Urol. doi:10.1016/j.eururo.2011.05.029 | Bertini, R. et al. The extent of tumour fat invasion affects survival in patients with renal cell carcinoma and venous tumour thrombosis. BJU Int. doi:10.1111/j.1464-410X.2010.09937.x 